\title{
Journal of Medical Virology
}

WILEY

\section{Co-circulation of three pathogenic hantaviruses: Puumala, Dobrava and Saaremaa in Hungary}

\begin{tabular}{|c|c|}
\hline Journal: & Journal of Medical Virology \\
\hline Manuscript ID: & JMV-09-1405.R1 \\
\hline Wiley - Manuscript type: & Research Article \\
\hline $\begin{array}{r}\text { Date Submitted by the } \\
\text { Author: }\end{array}$ & 21-Jul-2009 \\
\hline Complete List of Authors: & $\begin{array}{l}\text { Plyusnina, Angelina; Haartman Inst, University of Helsinki, Virology } \\
\text { Ferenczi, Emöke; B. Johan" National Center for Epidemiology } \\
\text { Rácz, Gábor; Harold W. Manter Laboratory of Parasitology, } \\
\text { University of Nebraska } \\
\text { Nemirov, Kirill; Swedish Institute for Infectious Disease Control } \\
\text { Lundkvist, Ake; Swedish Institute for Infectious Disease Control } \\
\text { Vaheri, Antti; Haartman Inst, University of Helsinki, Virology } \\
\text { Vapalahti, Olli; Haartman Inst, University of Helsinki, Virology } \\
\text { Plyusnin, Alexander; Haartman Inst, University of Helsinki, Virology }\end{array}$ \\
\hline Keywords: & HFRS; hantavirus; Puumala virus, Dobrava virus, Saaremaa virus \\
\hline
\end{tabular}

\section{scholarONE \\ Manuscript Central}


Fig. 1. Rodent collecting localities in Hungary (shown as full circles). Two localities (TR16 and TR17) that are discussed in the article are marked. Stars indicate localities from where hantavirus sequences have been recovered earlier (Scharninghausen et al., 1999; Jakab et al., 2007a, 2008). $540 \times 414 \mathrm{~mm}(72 \times 72 \mathrm{DPI})$ 


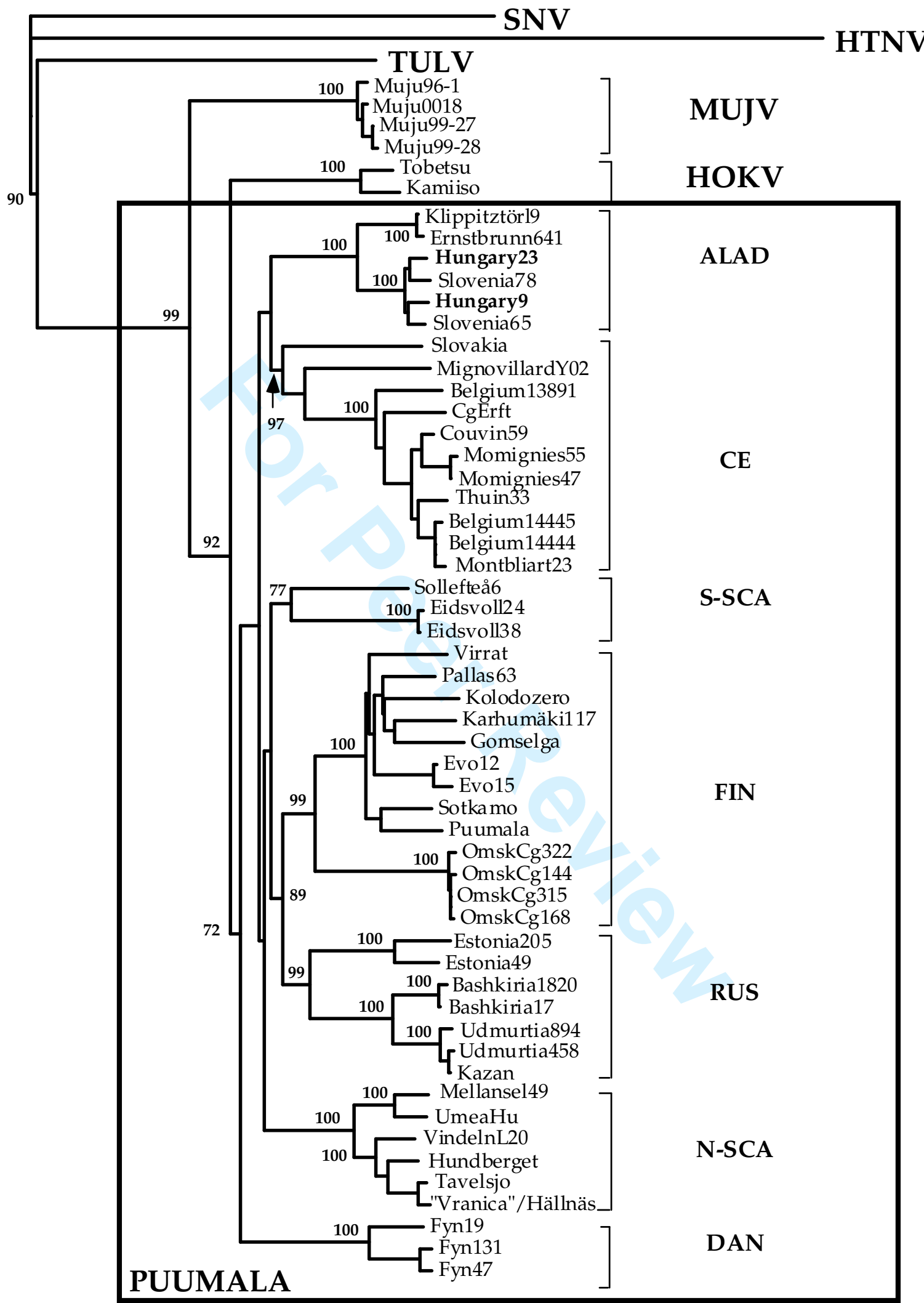

0.1 


\section{TULV}

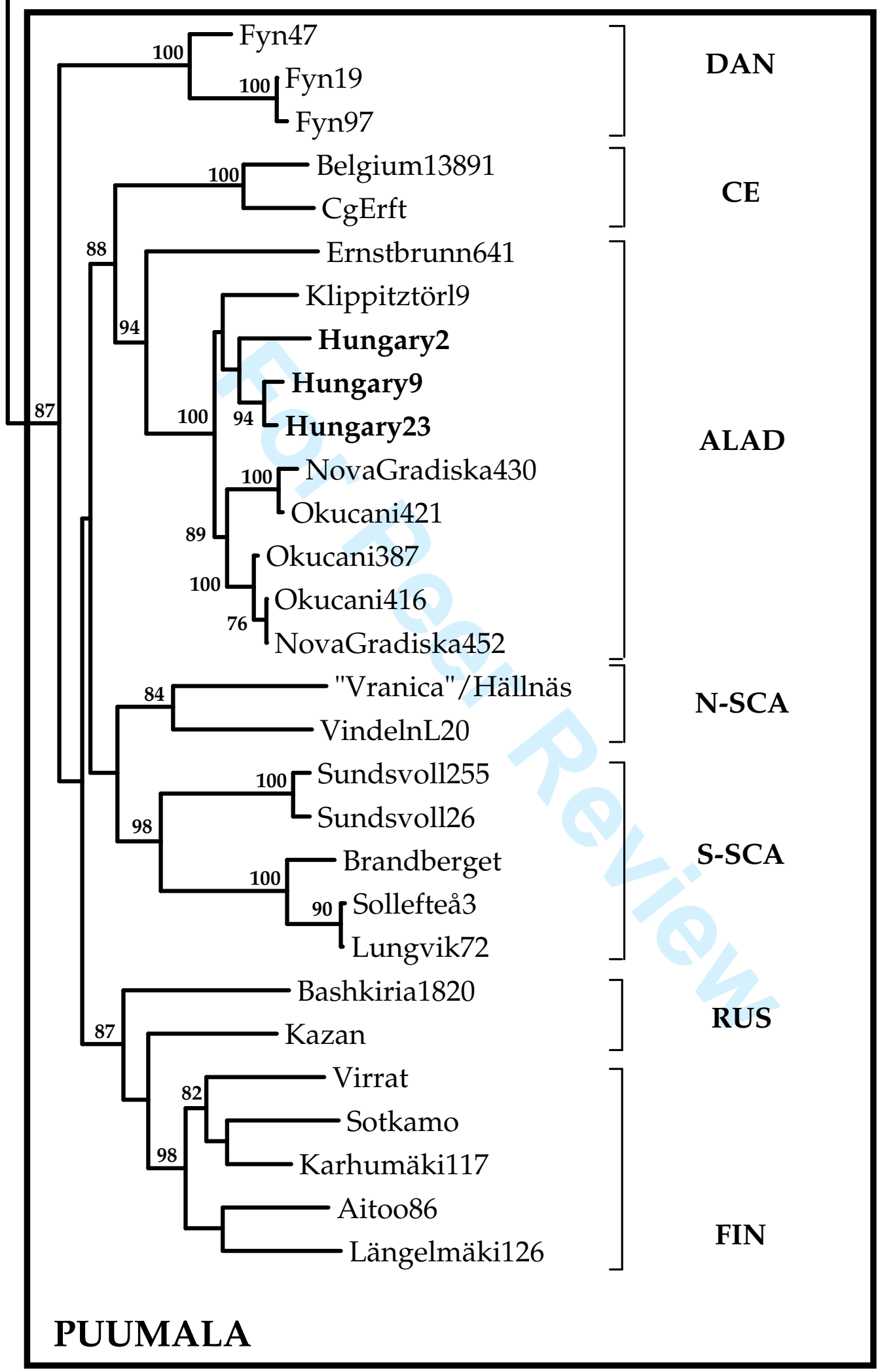

0.1 


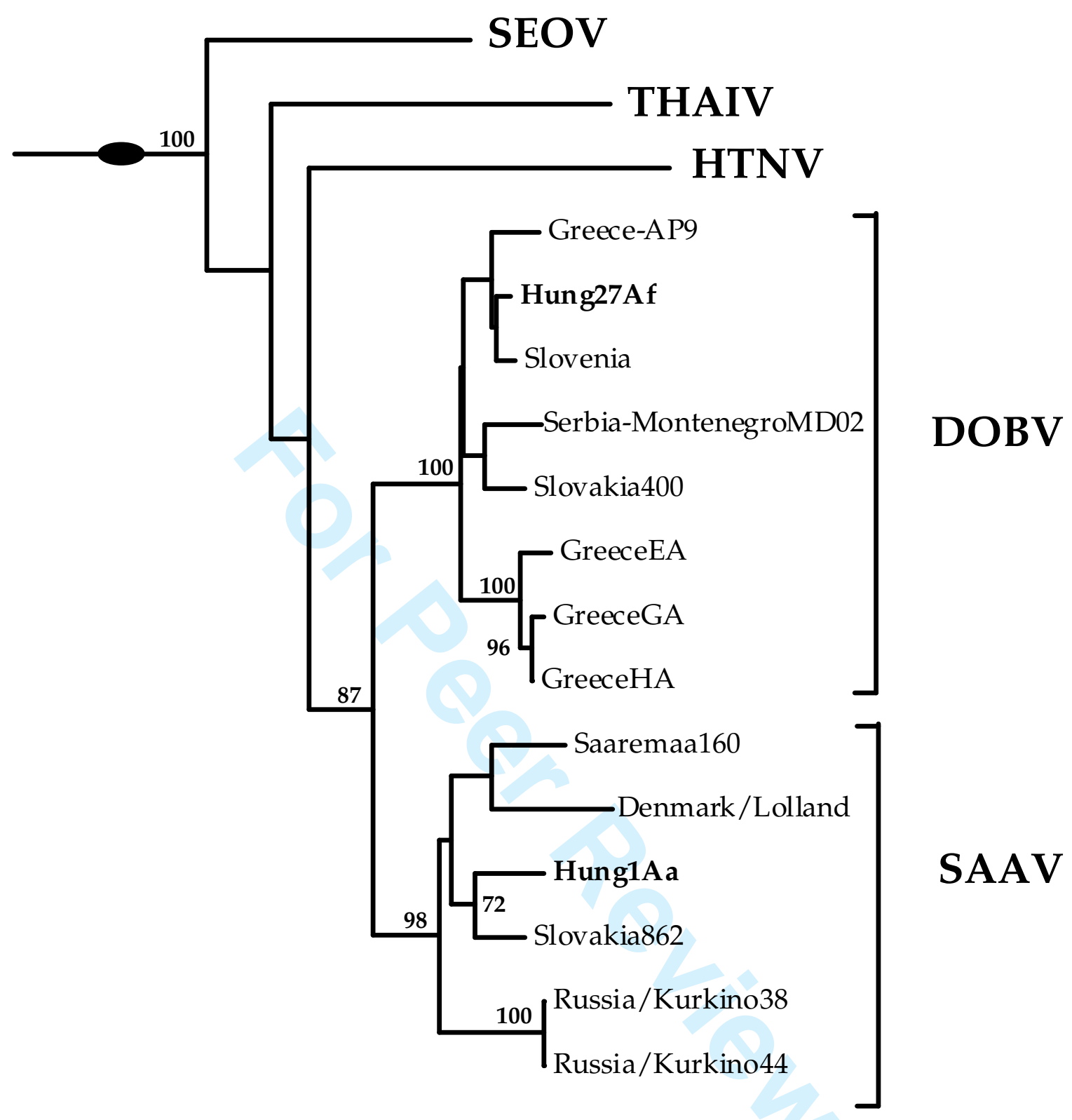




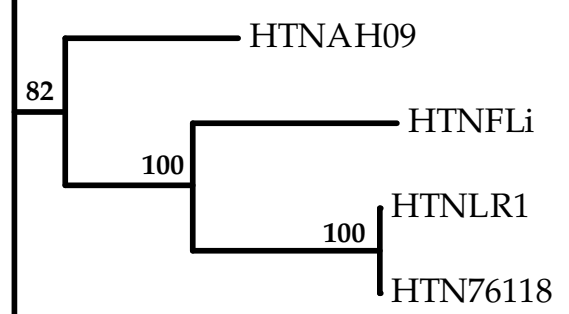

\section{GOUV} SEOR22 SEOSapporo

\section{SEOV}

THAIV

\section{HTNV} 0.1

SAAV

\section{DOBV}

THAIV
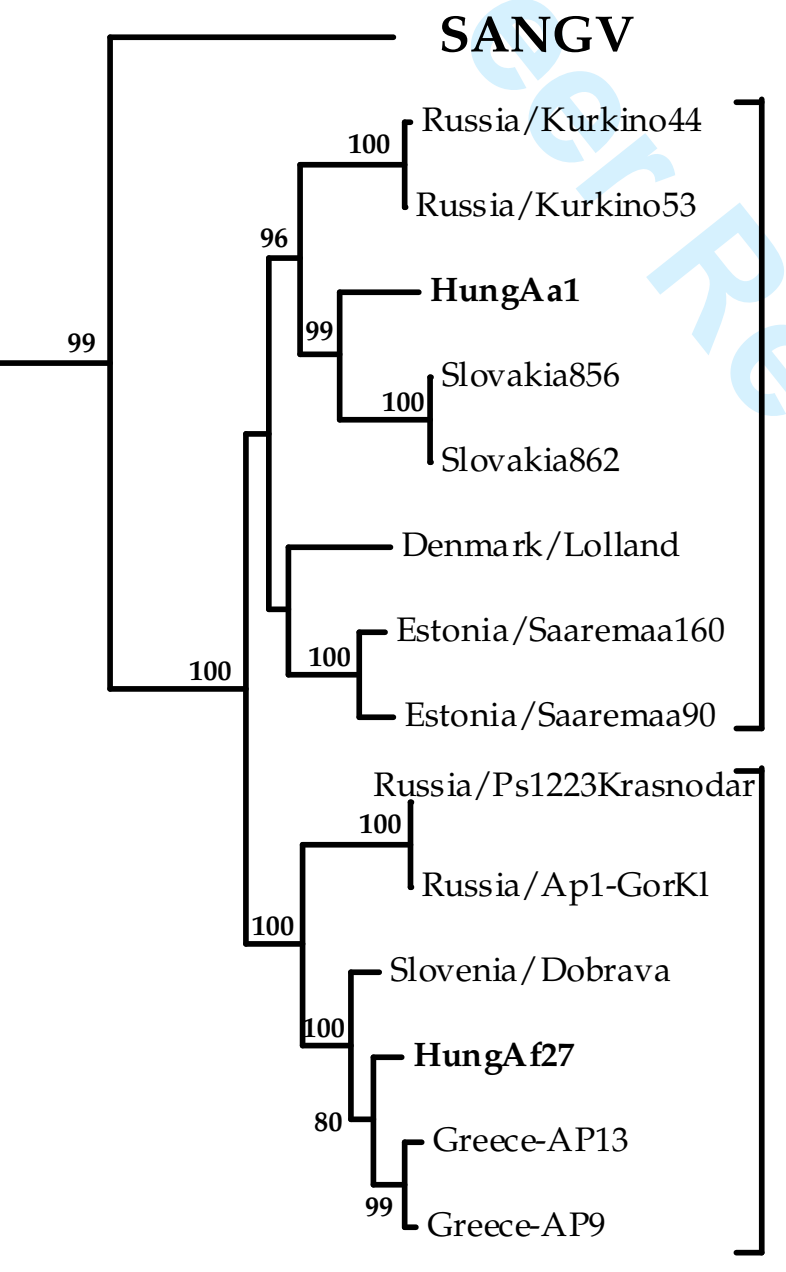


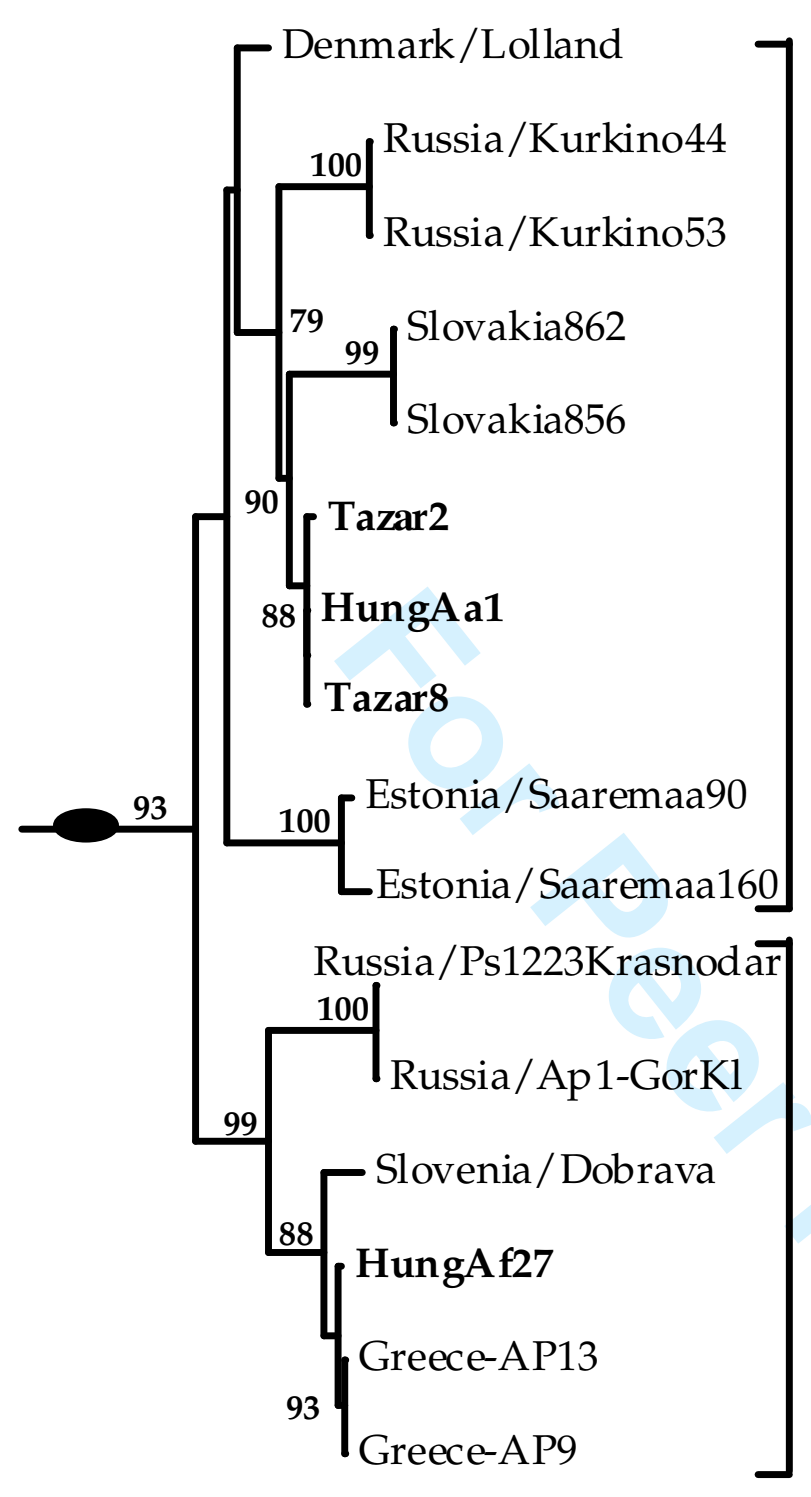

SAAV

\section{DOBV}

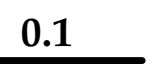




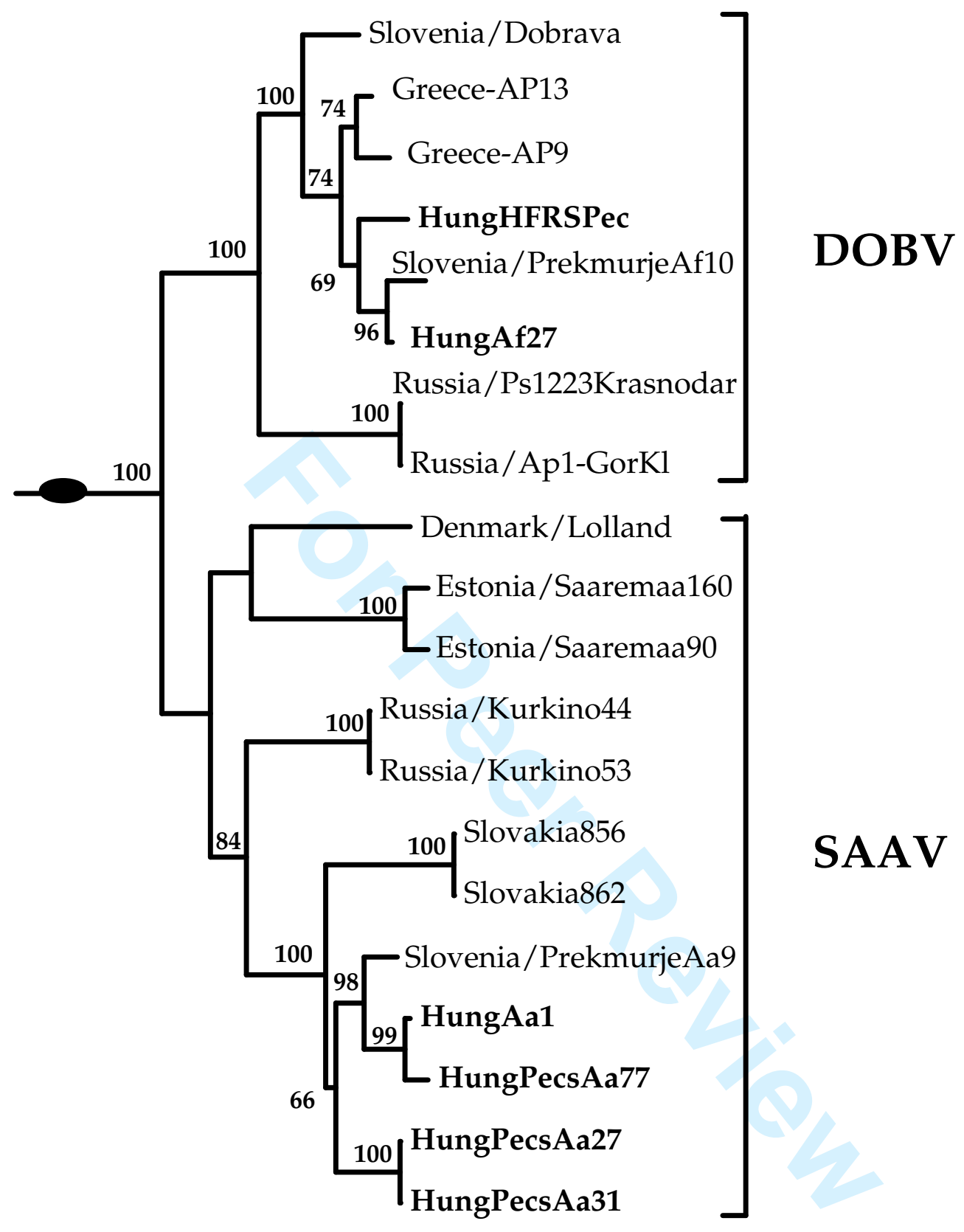

0.1 
3 4

5

7

9

10

11

12

13

14

24

\section{Co-circulation of three pathogenic hantaviruses: Puumala, Dobrava and Saaremaa in Hungary}

6 (Short title: Hantaviruses in Hungary)

23 Alexander Plyusnin

Correspondence to:

Department of Virology, Haartman Institute

University of Helsinki P. O. Box 21

FIN-00014, Finland
Angelina Plyusnina ${ }^{1}$, Emöke Ferenczi ${ }^{2}$, Gábor R. Rácz ${ }^{3}$, Kirill Nemirov ${ }^{4}$, Åke Lundkvist ${ }^{4}$, Antti Vaheri ${ }^{1}$, Olli Vapalahti ${ }^{1}$, and Alexander Plyusnin ${ }^{1}$

${ }^{1}$ Department of Virology, Infection Biology Research Program, Haartman Institute, University of Helsinki, Finland

2"B. Johan" National Center for Epidemiology, Budapest, Hungary

${ }^{3}$ Harold W. Manter Laboratory of Parasitology, University of Nebraska Lincoln, W529 Nebraska Hall, Lincoln, NE, U.S.A.

${ }^{4}$ Swedish Institute for Infectious Disease Control, Stockholm, Sweden 


\section{Page 9 of 30}

For J.Med.Virol.

27 Phone: +358 9 19126486; Fax: +358 919126491

28 E-mail: alexander.plyusnin@helsinki.fi 


\section{9 \\ ABSTRACT} 30

Hantaviruses (Bunyaviridae) cause hemorrhagic fever with renal syndrome (HFRS) in Eurasia and hantavirus (cardio)pulmonary syndrome (HCPS) in the Americas. HFRS is caused by Hantaan (HTNV), Seoul (SEOV), Dobrava (DOBV), Saaremaa (SAAV) and Puumala (PUUV) viruses. Of those, only HTNV is not present in Europe. In recent years, hantaviruses, described in other parts of Europe, were also detected at various locations in Hungary. To study the genetic properties of Hungarian hantaviruses in detail, sequences of the viral $S$ and $M$ segments were recovered from bank voles (Myodes glareolus), yellow-necked mice (Apodemus flavicollis) and striped field mice (Apodemus agrarius) trapped in the Transdanubian region. As expected, the sequences recovered belonged, respectively, to PUUV (two strains), DOBV (one strain) and SAAV (one strain). On phylogenetic trees two new Hungarian PUUV strains located within the well-supported Alpe-Adrian (ALAD) genetic lineage that included also Austrian, Slovenian and Croatian strains. Analysis of the Hungarian SAAV and DOBV genetic variants showed host-specific clustering and also geographical clustering within each of these hantavirus species. Hungarian SAAV and DOBV strains were related most closely to strains from Slovenia (Prekmurje region). This study confirms that multiple hantaviruses can co-circulate in the same locality and can be maintained side-by-side in different rodent species.

Key words: HFRS; hantavirus; Puumala virus, Dobrava virus, Saaremaa virus. 
For J.Med.Virol.

52 53

\section{INTRODUCTION}

Hantaviruses (genus Hantavirus, family Bunyaviridae) cause hemorrhagic fever with renal syndrome (HFRS) in Eurasia and hantavirus (cardio)pulmonary syndrome (HCPS) in the Americas (for reviews, see Schmaljohn and Hjelle, 1997; Vapalahti et al., 2003). HFRS is caused by Hantaan virus (HTNV), Seoul virus (SEOV), Dobrava-Belgrade virus (DOBV), Saaremaa virus (SAAV) and Puumala virus (PUUV). Sin Nombre virus (SNV), Andes virus and related hantaviruses are the main causative agents of severe HCPS. Hantaviruses are host-specific, and thus their distribution correlates most often with the geographical distribution of the natural host species, rodents and insectivores. Transmission of hantaviruses to humans occurs by inhalation of infested aerosols of rodent excreta. Whether hantaviruses carried by insectivores can infect humans and cause any disease remains to be seen.

Of HFRS-causing hantaviruses, only HTNV is not present in Europe [Vapalahti et al., 2003]. PUUV, carried by bank voles (Myodes glareolus), is the most widely distributed European hantavirus. It causes a mild form of HFRS (also called nephropathia epidemica, NE) in Northern and Central Europe, European part of Russia and in the Alpe-Adrian region. DOBV, harbored by yellow-necked field mice (Apodemus flavicollis), is the most severe European hantavirus pathogen; it is associated with HFRS mostly in the Balkans [Papa et al., 1998; Avsic-Zupanc et al., 1999]. SAAV, carried by striped field mice (Apodemus agrarius), causes mild HFRS resembling NE; the virus is found in the Baltics, Central Europe and European part of Russia [Lundkvist et al., 1998; Plyusnin et al., 1999; Golovljova et al., 2000; Sibold et al., 2001]. SEOV, carried by Norway rats (Rattus norvegicus), is so far found in 
For J.Med.Virol.

76 France and Belgium [Heyman et al., 2004, 2009]. Apart from laboratory outbreaks

77 caused by SEOV-infected rats kept in captivity, no human cases in Europe have

78 been reported in connection to this hantavirus.

79

80

81

82

83

84

85

86

87

88

89

90

91

92

93

94

95

96

97

98

99

HFRS has been described in Hungary prior to the identification of the causative agents, the hantaviruses [Trencsenyi and Keleti, 1981]. Until 1985, human HFRS cases were diagnosed initially based on medical symptoms and epidemiology; these were later confirmed using serological tests [Takenaka et al., 1985; Faludi and Ferenczi 1995]. With the advent of DNA and RNA sequencing several hantaviruses have been identified in Hungary [Scharninghausen, 1999, Ferenczi 2003, Jakab 2007a, 2008].

On average, there are 20-25 laboratory-confirmed human hantavirus infections in Hungary each year. The fatality rate associated with clinical infections dropped from approximately $6 \%$ in the 1950 s to $2.5 \%$ in recent years. In case a physician suspects HFRS, further serological tests are performed at the National Center for Epidemiology (NCE). Serological diagnosis is based primarily on immunofluorescence assay (IFA) and secondarily on enzyme-immunoassay (EIA). Compared to the number of hospitalized patients, hantavirus seroprevalence is much higher in Hungary, approximately 10\% [Ferenczi et al., 2005]. These controversial data might be explained by the low HFRS awareness of the medical community and by the undiagnosed mild forms of the disease.

The aim of this study was to recover hantavirus sequences from tissue samples of rodents known as hatural hosts for Puumala, Dobrava and Saaremaaa viruses, perform their detailed (phylo)genetic characterization, assign them to proper taxa and specify unequivocally the rodent hosts involved. 


\section{Page 13 of 30}

For J.Med.Virol.

100

3

4

5

6

7

8

9

10

11

12

13

14

15

16

17

18

19

20

21

22

23

24

25

26

27

28

29

30

31

32

33

34

35

36

37

38

39

40

41

42

43

44

45

46

47

48

49

50

51

52

53

54

55

56

57

58

59

60

John Wiley \& Sons 
For J.Med.Virol.

101

102

103

104

105

106

107

108

109

110

111

112

113

114

115

116

117

118

119

120

121

122

123

124

\section{MATERIALS AND METHODS}

Trapping of rodents. Small mammals were collected as part of a larger research project on studying the distribution of hantaviruses in Hungary. The broader area, the southwestern part of Hungary, was selected based on the high incidence rate of human hantavirus cases (Fig. 1). Zala county with only $3 \%$ of Hungary's total population gives $23 \%$ of HFRS cases in the country. In 2000, selected trapping localities were chosen to survey wild mammals as reservoirs of hantaviruses in forest habitats. The primary factor in selecting specific sites was the forest area, but several other characteristics of the plant community (e.g. disturbance level and plant species composition) correlated with patch size. Rodents were trapped using Sherman type live capture traps [Mills et al., 1995] set in the evening and checked in the morning. At each locality trapping was continued until sufficient numbers of animals (usually around 40-50) were captured. Animals were identified, aged, sexed and their conditions were recorded. Euthanized rodents were dissected; blood and tissue samples - lung, spleen, kidney, heart - were removed for further analysis. Tissues were frozen immediately in liquid nitrogen. Upon arrival at the Virology Department of the NCE tissue samples were transferred to $-80^{\circ} \mathrm{C}$ freezers for long-term storage. Skeletal materials of the collected animals were deposited as voucher specimens at the Mammal Collection of the Museum of Southwestern Biology, University of New Mexico.

Screening of rodent samples. Sera collected from rodents were screened initially for the presence of antibodies to hantaviruses by commercially available kits as described previously [Ferenczi et al., 2003]. Briefly, HTNV antigen-coated 
For J.Med.Virol.

high density particle agglutination (HDPA) kit (Korean Green Cross) was used according the protocol of the WHO Collaborating Center for Virus Reference and Research (HFRS), Institute for Viral Diseases, Korea University, Seoul, South Korea. To perform indirect immunofluorescent assay (IFA), HTNV-, PUUV- and SEOVspecific tests (Progen Biotechnik, GMBH, Germany) with FITC-conjugated rabbit anti-mouse immunoglobulin (DAKO, A/S, Denmark) were used. For the detection of HTNV- and PUUV-specific antibodies by enzyme immunoassay (EIA), IgG and IgM kits (Progen Biotechnik) were applied.

Twenty five out of 37 serum samples were tested additionally on EIA plates containing recombinant antigens for PUUV, DOBV and SEOV viruses [Elgh et al., 1997]. Lung tissue samples from seropositive rodents were screened further for hantavirus nucleocapsid $(\mathrm{N})$ protein antigen by immunoblotting as described earlier [Plyusnin et al., 1995].

Reverse transcription - polymerase chain reaction (RT-PCR) and sequencing. RNA was extracted from lung tissue samples using the Tripure reagent (Boehringer Mannheim) following recommendations of the manufacturer. RNA was then subjected to RT-PCR to recover partial S segment and $M$ segment sequences (sequences of primers and other experimental details are available upon request). PCR amplicons were gel-purified with QIAquick Gel Extraction -kit (QIAGEN) and sequenced directly using ABI PRISMTM Dye Terminator sequencing kit (Perkin Elmer/ABI, NJ). PUUV genome sequences described in this paper have been deposited to the GenBank sequences database under accession numbers FN37782125. DOBV and SAAV genome sequences described in this paper have been deposited to the GenBank sequences database under accession numbers FN377826-29. 
For J.Med.Virol.

149 Mitochondrial DNA (mtDNA) analysis was performed as described earlier

150 [Nemirov et al., 2002]. Briefly, DNA was extracted from lung tissue samples using 151 the Tripure reagent. A 427 nt-long PCR-product from the D-loop-encoding region 152 was amplified with primers 5'-CCACCATCAGCACCCAAAGCTG-3' and 5'153 CTGAAGTAAGAACCAGATGTCTG-3'. The product was purified from the gel and 154 subjected to direct sequencing. For comparison, mtDNA sequences of A. agrarius and 155 A. flavicollis were retrieved from the GenBank nucleotide databases.

156 Phylogenetic analysis. Multiple nucleotide alignments were prepared 157 manually using SeqApp 1.9a169 sequence editing program. Phylogenetic analysis 158 was performed using the PHYLIP program package [Felsenstein, 1993]. 500 159 bootstrap replicates (Seqboot program) were submitted to the distance matrice 160 algorithm (Dnadist program), with maximum likelihood model for nucleotide 161 substitutions); distance matrices were analysed with the Fitch-Margoliash tree-fitting 162 algorithm (Fitch program); the bootstrap support values were calculated with the 163 Consense program. Hantavirus sequences used for comparison were recovered from 164 the GenBank. 
For J.Med.Virol.

165

166

167

168

169

170

171

172

173

174

175

176

177

178

179

180

181

182

183

184

185

186

187

188

\section{RESULTS}

Prescreening of rodents. Rodent candidates for further RT-PCR and sequencing were pre-screened for hantavirus antibodies by HDPA, IFA and EIA. From more than 300 rodents, 37 were found positive or suspected positive: 8 bank voles (Myodes glareolus) and 29 Apodemus mice (18 A. agrarius, 2 A. flavicollis, 9 unspecified Apodemus spp.). Positive M. glareolus were from the locality TR17, positive Apodemus mice from localities TR16 and TR17 (Fig. 1).

Genetic analysis of PUUV strains. From eight pre-selected (Ab-positive) bank voles, six were found positive for PUUV N-Ag in immunoblotting. Of those, three appeared also positive for PUUV genome when tested by RT-PCR; all three animals were from the same locality, TR-17. Three wild-type PUUV strains (that were not isolated) were designated PUUV $/ \mathrm{Mg} 2 /$ HungaryTR17/00, PUUV/Mg9/HungaryTR17/00, and PUUV/Mg23/HungaryTR17/00, or Hung2, Hung9, and Hung23, for short. For two strains, Hung9 and Hung23, almost complete sequences of the $S$ segment have been recovered in a set of (semi)-nested PCRs. For the strain Hung2, only partial sequence of the coding region (nt 191 to 1190) was recovered. It differed by two silent nucleotide substitutions from the corresponding sequence of strain Hung9 and was excluded from further analyses. Two other sequences included 5'-noncoding region (NCR) of $42 \mathrm{nt}$ and the open reading frame (ORF) for the N protein of 433 aa followed by 499 or 442 nt from 3'NCR (for strains Hung9 and Hung23, respectively). The 5'-NCR sequences were identical (the first $22 \mathrm{nt}$ originated from the primer sequence and thus were not determined directly); the coding regions differed by 34 substitutions (identity of 
For J.Med.Virol.

$18997.4 \%$ ); the 3'-NCR sequences differed at 28 positions (identity of 95.9\%). Deduced N

190 protein sequences differed by one substitution only (identity of $99.8 \%$ ) and carried

191 signature residues V236 and P257 shared also by PUUV strains from neighbouring

192 Austria [Plyusnina et al., 2006] and Slovenia [Avsic-Zupanc et al., 2007]. Partial M

193 segment sequences (nt 2161-2570, primers excluded) of strains Hung2, Hung9, and

194 Hung23 were recovered as well; they appeared 94.4-96.1\% identical to each other.

195 The encoded partial sequences of the Gc protein (136 aa long) were 97.8-99.3\%

196 identical. Hungarian M segment/Gc protein sequences were most closely related to

197 the corresponding sequences from Austrian [Plyusnina et al., 2006] and Croatian

198 [Cvetko et al., 2005] PUUV strains (corresponding sequences from Slovenian strains

199 are not available).

200 On the phylogenetic tree based on the complete S segment coding region, the 201 two Hungarian PUUV strains were located within the well-supported Alpe-Adrian 202 (ALAD) genetic lineage that included also Austrian and Slovenian strains (Fig. 2A). 203 Within this lineage, Hungarian and Slovenian strains shared the most recent 204 common ancestor (TMRCA) while Austrian strains formed a sister taxon to this 205 quartet. Six other PUUV lineages can be recognized: (1) Central European (CE); (2) 206 South Scandinavian (S-SCA); (3) Finnish (FIN); (4) Russian (RUS); (5) North 207 Scandinavian (N-SCA); and (6) Danish (DAN). PUUV shared more ancient common 208 ancestors (MACAs) with Hokkaido virus (HOKV) and then with Muju virus 209 (MUJV) associated with Myodes rufocanus and Myodes regulus, respectively. The 210 phylogenetic tree based on the partial M segment sequence showed similar topology 211 (Fig. 2B): Hungarian strains were located within the ALAD lineage which, in this 
For J.Med.Virol.

212 case, included also strains from Austria and Croatia. Other PUUV lineages (CE, S213 SCA, FIN, RUS, N-SCA and DAN) were presented as well.

214 Genetic analysis of SAAV and DOBV strains. From 29 pre-screened 215 Apodemus mice (18 A. agrarius, 2 A. flavicollis, 9 - unspecified); six were found 216 positive for DOBV/SAAV N-Ag in immunoblotting. Of those, only two were 217 positive also in the RT-PCR test. Analysis of mtDNA sequences showed that one 218 mouse (\#1, from locality TR-16) belonged to the species A. agrarius, another (\#27 219 from locality TR-17) was A. flavicollis. Partial S and M segment sequences were 220 recovered from both rodents using DOBV/SAAV-specific primers and subjected to 221 phylogenetic analysis. On phylogenetic trees (Fig. 3A-B), the sequence recovered 222 from $A$. agrarius mouse was placed with SAAV sequences (all recovered from 223 striped field mice) while the sequence recovered from A. flavicollis mouse co224 localized with DOBV sequences, originated from either yellow-necked mice or 225 human HFRS cases. Although the topologies of the trees based on partial M and S 226 segment sequences were quite similar, the M-tree showed a somewhat better 227 resolution, i.e. higher bootstrap support values for SAAV- and DOBV-clades. Based 228 on these data, novel Hungarian hantavirus strains were designated 229 SAAV/Aa1/Hungary TR16/00 and DOBV/Af27/HungaryTR17/00 or HungAa1 230 and HungAf27, for short.

231 New Hungarian SAAV and DOBV sequences were compared to previously 232 published ones recovered from A. agrarius trapped in Taszár [spelled as "Tazar" in 233 the original publication], eastern Hungary [Scharninghausen et al., 1999]. The 234 analysis was restricted to the overlapping part of the $\mathrm{S}$ segment sequence recovered 235 in two studies: nt 707 to 935 . This region of two Taszár strains appeared most 
For J.Med.Virol.

236

237

238

239

240

241

242

243

244

245

246

247

248

249

250

251

252

253

254

255

256

257

258

259

closely related to the corresponding region of the SAAV strain HungAa1: only one (silent) nucleotide substitution was observed between these sequences (identity of 99,6\%). In contrast, the sequence of the DOBV strain HungAf27 differed from Taszár sequences at $29-30$ positions (identity of $86.9-87.3 \%$ ). Phylogenetic analysis showed TMRCA for the two Taszár strains and the strain HungAa1 (Fig. 3C). This trio was placed within the SAAV clade and shared MACA with SAAV strains from Slovakia. These data suggested that the two Taszár strains belong to SAAV.

New Hungarian SAAV and DOBV sequences were compared also to the previously published sequences recovered from A. agrarius trapped in the Görcsöny and Sármellék areas and from an HFRS patient from the University hospital of Pécs (all in the Transdanubian region) [Jakab et al., 2007a,b]. Since, in this case, the analysis was restricted to shorter sequences of the $S$ segment recovered by these authors (nt 410 to 800 ), it became possible to include also partial SAAV and DOBV sequences originated from Prekmurje region of neighbouring Slovenia [AvsicZupanc et al., 2000].

Direct sequence comparison showed that Hungarian SAAV strain HungAa1 was most closely related to Hungarian strain Pecs/77Aa from Sármellék (sequence identity 99.5\%) and to Slovenian SAAV strain Aa9 from Prekmurje (97.9\%). Sequence of our DOBV strain HungAf27 was most closely related to the corresponding region of Slovenian DOBV strain from Prekmurje (99.2\%) and then to Hungarian HFRS-associated strain (92.0\%). On the phylogenetic tree (Fig. 3D) all four Hungarian A. agrarius originated sequences, as expected, were located within the SAAV clade. The new strain HungAa1 shared TMRCA with the strain PecsAa77 and this couple shared the MACA with Slovenian strain Aa9 from Prekmurje. 


\section{Page 21 of 30}

For J.Med.Virol.

260 Similarly, our DOBV strain HungAf27 shared TMRCA with Slovenian DOBV strain

261 Af10 from Prekmurje; they, in turn, shared MACA with the Hungarian HFRS-

262 associated strain. These results demonstrated host-specific clustering of Hungarian

263 SAAV and DOBV genetic variants and, within each of these hantavirus species, also

264 geographical clustering. 


\section{DISCUSSION}

The results of this study demonstrate co-circulation of all three major European hantavirus pathogens in Hungary: PUUV, DOBV and SAAV. They are in line with earlier observations on human HFRS cases in this country [Ferenczi et al., 2005; Jakab et al., 2007a] and recovery of hantavirus sequences from rodents [Scharninghausen et al., 1999; Ferenczi et al., 2003; Jakab et al., 2007a]. It should be emphasized that while SAAV in Hungarian striped field mice has been already reported (albeit under the name of Dobrava virus - see below), the first unequivocal evidence for PUUV and DOBV in Hungarian rodents, species M. glareolus and A. flavicollis, respectively, is presented in this paper.

Rodents used in genetic studies were selected in two steps. First, pre-selection by HDPA, EIA and IFA to detect hantavirus antibodies was performed. On that stage, not only clearly Ab-positive but also borderline voles and mice were selected for further analyses. This could explain the observed differences between the results of antibody detection and the N-Ag detection, due to pre-selection not only clearly Ab-positive but also voles and mice giving the borderline results. In turn, the relatively low RT-PCR positivity of N-Ag-positive rodents could be due to partial degradation of target RNA in tissue samples that were subjected to several thawingfreezing cycles (to take material for other analyses) before RNA extraction.

Phylogenetic analysis of Hungarian PUUV strains placed them within the ALAD genetic lineage that includes also strains from neighbouring Slovenia, Croatia and Austria. This presents a good example of geographical clustering of PUUV genetic variants, a phenomenon first discovered more than a decade ago [Plyusnin 
For J.Med.Virol.

et al., 1994]. Phylogenies that included new Hungarian PUUV sequences confirmed two interesting earlier observations [Plyusnina et al., 2006]. First, within the ALAD lineage, two Austrian M-sequences are not monophyletic. One of the strains, Klipp9, is clustering together with Croatian and Hungarian strains suggesting that $\mathrm{M}$ and $\mathrm{S}$ segments of this strain might have different evolution history, i.e. this strain is a reassortant one. Second, ALAD and CE lineages and RUS and FIN lineages share MACAs thus suggesting somewhat closer relationships within these two pairs of lineages that could be rooted to the last postglacial recolonization of these areas of Europe.

The analyses of Hungarian SAAV and DOBV sequences confirmed that genetic variants of these hantavirus species show the host-specific clustering. Indeed, all sequences recovered from $A$. agrarius belonged to SAAV while those from $A$. flavicollis belonged to DOBV. Thus previously reported sequences from Hungarian A. agrarius [Scharninghausen et al., 1999; Jakab et al., 2007a] should be re-classified as SAAV strains. It seems that only the sequence recovered from human HFRS case in Pécs [Jakab et al., 2007b] presents bona fide DOBV. As mentioned above, for several years there was a controversy in taxonomy and hence terminology of SAAV. When first discovered, this Dobrava-like virus was erroneously considered a genetic variant of DOBV carried by the striped field mice [Plyusnin et al., 1997; Nemirov et al., 1999]. It took several years to realize that this is in fact a distinct hantavirus species [Brus-Sjölander et al., 2002; Nemirov et al., 2002], the view that is now shared by the International Committtee for Virus Taxonomy [www.ictvonline.org].

Another important finding of this study is that multiple hantavirus types can be maintained at the same locality by their corresponding rodent hosts. The direct 
For J.Med.Virol.

transmission mode and the high level of host specificity of the hantaviruses make these temperate-zone viruses less affected by host biodiversity [Ruedas et al., 2004]. Instead, the population density and size are the main factors driving the disease temporal dynamics.

Of the four European hantavirus pathogens known so far, only SEOV has not been found in Hungary, albeit serological evidence showed its causative role at least in one case [Ferenczi, unpublished data]. Direct detection of this virus is rather difficult because its primary hosts, Norway rats Rattus norvegicus, would not be caught by the traps used. Similar observation have been made in neighbouring Slovenia [Avsic-Zupanc et al., 2002, 2007], Slovakia [Sibold et al., 2001] and Croatia [Cvetko et al., 2004]. Our unpublished serological data on clinical cases confirm that both PUUV-like and DOBV/SAAV-like HFRS cases occur in Hungary. Cocirculation of three distinct hantaviruses that could cause HFRS of different severity, including life-threatening infections, calls for improvements in both surveillance and diagnostics.

Acknowledgements. The work was supported by The Academy of Finland and Sigrid Juselius Foundation (Finland). Rodent survey was funded by grants from Hungarian Ministry of Health (ETT 1998-1999. Rec. No.: 144/98), and Hungarian Ministry of Environment (II. OKTKP id. No.:020837-01/2000). Field research equipment was partially provided by funding from the Centers for Disease Control and Prevention through a cooperative agreement with Dr. Terry L. Yates. The authors are grateful to Borbala Juhasz, Marta Csire, Kaposi Tamasné and AnnChristin "Anci" Verlemyr for excellent technical assistance. 


\section{Page 25 of 30}

For J.Med.Virol. 
Avsic-Zupanc T, Petrovec M, Furlan P, Kaps R, Elgh F, Lundkvist Å. 1999. Hemorrhagic fever with renal syndrome in the Dolenjska region of Slovenia-A 10-year survey. Clin Infect Dis 28: 860-865.

342 Avsic-Zupanc T, Nemirov K, Petrovec M, Trilar T, Poljak M, Vaheri A, Plyusnin A. 343 2000. Genetic analysis of wild-type Dobrava hantavirus in Slovenia: co-existence of two 344 distinct genetic lineages within the same natural focus. J Gen Virol 81:1747-1755.

345 Avsic-Zupanc T, Petrovec M, Dun D, Plyusnina A, Lundkvist Å, Plyusnin A. 2007.

346 Puumala hantavirus in Slovenia: analyses of $\mathrm{S}$ and $\mathrm{M}$ segment sequences recovered 347 from patients and rodents. Virus Res 123: 204-210.

348 Brus-Sjölander K, Golovljova I, Plyusnin A, Lundkvist Å. 2002. Serological divergence 349 of Dobrava and Saaremaa hantaviruses: evidence for two distinct serotypes. Epidemiol 350 \& Infection 128:99-103.

351 Elgh F, Lundkvist A, Alexeyev OA, Stenlund H, Avsic-Zupanc T, Hjelle B, Lee HW, 352 Smith KJ, Vainionpää R, Wiger D, Wadell G, Juto P. 1997. Serological diagnosis of 353 hantavirus infections by an enzyme-linked immunosorbent assay based on detection of 354 immunoglobulin $\mathrm{G}$ and $\mathrm{M}$ responses to recombinant nucleocapsid proteins of five viral 355 serotypes. J Clin Microbiol 35:1122-1130.

356 Faludi G, Ferenczi E. 1995. Seriologically verified Hantavirus infections in Hungary, 357 Acta Microbiol Immunol Hung 42:419-426.

358 Felsenstein 1993. PHYLIP (Phylogeny Inference Package) version 3.5c. Distributed by 359 the author. Department of Genetics, University of Washington, Seattle.

360 Ferenczi E, Rácz G, Szekeres J, Balog K, Tóth E, Takács M, Csire M, Mezey I, Berencsi G, 361 Faludi G. 2003. [New data for the public health importance of hantaviruses in 362 Hungary]. [Article in Hungarian] Orv Hetil 144:467-474.

363 Ferenczi E, Rácz G, Faludi G, Czeglédi A, Mezey I, Berencsi G. 2005. Natural foci of 364 classical and emerging viral zoonoses in Hungary. In: Berencsi G, Khan AS, Halouska J, 365 editors. NATO Science Series: Emerging Biological Threat. IOS Press, p. 43-49. 
366 Golovljova I, Vasilenko V, Prukk T, Brus Sjolander K, Plyusnin A, Lundkvist Å. 2000.

367 Puumala and Dobrava hantaviruses causing hemorrhagic fever with renal syndrome in 368 Estonia. Eur J Clin Microbiol Infect Dis 19:968-969.

369 Golovljova I, Brus Sjölander K, Lindegren G, Vene S, Vasilenko V, Plyusnin “A, 370 Lundkvist Å. 2002. Hantaviruses in Estonia. J Med Virol 68:589-598.

371 Heyman P, Plyusnina A, Berny Ph, Cochez C, Artois M, Zizi M, Pirnay JP, Plyusnin A. 372 2004. Seoul hantavirus in Europe: first demonstration of the virus genome in wild $R$. 373 norvegicus captured in France. Eur J Clin Microbiol Infect Dis. 23:711-717.

374 Heyman P, Baert K, Plyusnina A, Cochez C, Lundkvist Å, Van Esbroeck M, Goossens E, 375 Vandenvelde C, Plyusnin A, Stuyck J. 2009. Serological and genetic evidence for the 376 presence of Seoul hantavirus in Rattus norvegicus in the Flanders, Belgium. Scand J 377 Infect Dis 41:51-56.

378 ICTV website: $\underline{w w w . i c t v o n l i n e . o r g}$

379 Jakab F, Horváth G, Ferenczi E, Sebok J, Varecza Z, Szucs G. 2007a. Detection of 380 Dobrava hantaviruses in Apodemus agrarius mice in the Transdanubian region of 381 Hungary. Virus Res 128:149-152.

382 Jakab F, Sebok J, Ferenczi E, Horváth G, Szucs G. 2007b. First detection of Dobrava 383 hantavirus from a patient with severe haemorrhagic fever with renal syndrome by 384 SYBR Green-based real time RT-PCR. Scand J Infect Dis 39:902-906.

385 Jakab F, Horváth, Ferenczi E, Sebok J, Szucs, G. 2008. First detection of Tula 386 hantaviruses in Microtus arvalis voles in Hungary. Arch Virol 153: 2093-2096.

387 Lundkvist $\AA$, Vasilenko V, Golovljova I, Plyusnin A, Vaheri, A. 1998. Human Dobrava 388 hantavirus infections in Estonia. Lancet 352:369.

389 Mills JN, Yates TL, Childs JE, Parmenter RR, Ksiazek TG, Rollin PE, Peters CJ. 1995. 390 Guidelines for working with rodents potentially infected with hantavirus. J 391 Mammalogy 76:716-722.

392 Nemirov K, Vapalahti O, Lundkvist A, Vasilenko V, Golovljova I, Plyusnina A, 393 Niemimaa J, Laakkonen J, Henttonen H, Vaheri A, Plyusnin A. 1999. Isolation and 394 characterization of Dobrava hantavirus carried by the striped field mouse (Apodemus 395 agrarius) in Estonia. J Gen Virol 80:371-379. 
Nemirov K, Henttonen H, Vaheri A, Plyusnin A. 2002. Phylogenetic evidence for host switching in the evolution of hantaviruses carried by Apodemus mice. Virus Res 90: 207215, 2002. (Corrigendum: Virus Res 92:125-126, 2003).

Papa A, Johnson AM, Stockton PC, Bowen MD, Spiropoulou CF, Alexiou-Daniel S, Ksiazek TG, Nichol ST, Antoniadis A. 1998. Retrospective serologic and genetic study of the distribution of hantaviruses in Greece. J Med Virol 55: 321-327.

Plyusnin A, Vapalahti O, Ulfves K, Lehväslaiho H, Apekina N, Gavrilovskaya I, Blinov V, Vaheri A. 1994. Sequences of wild Puumala virus genes show a correlation of genetic variation with geographic origin of the strains. J Gen Virol 75:405-409.

Plyusnin A, Cheng Y, Vapalahti O, Pejcoch M, Unar J, Jelinkova Z, Lehväslaiho H, Lundkvist $\AA$, Vaheri, A. 1995. Genetic variation in Tula hantaviruses:; sequence analysis of the $S$ and $M$ segments of strains from Central Europe. Virus Res 39: 237-250.

Plyusnin A,Vapalahti O, Vasilenko V, Henttonen H, Vaheri A. 1997. Dobrava hantavirus in Estonia: does the virus exist throughout Europe? Lancet 349:1369-1370.

Plyusnin A, Nemirov K, Apekina N, Plyusnina A, Lundkvist Å, Vaheri A. 1999. Dobrava hantavirus in Russia. Lancet 353:207.

Plyusnina A, Aberle S, Aberle J, Plyusnin A. 2006. Genetic analysis of Puumala hantavirus strains from Austria. Scand J Infect Dis 38: 512-519.

Ruedas LA, Salazar-Bravo J, Tinnin DS, Armién B, Cáceres L, García A, Díaz MA, Gracia F, Suzán G, Peters CJ, Yates TL, Mills, JM. 2004. Community ecology of small mammal populations in Panamá following an outbreak of Hantavirus pulmonary syndrome. J Vector Ecol 29:177-191.

Scharninghausen JJ, Meyer H, Pfeffer M, Davis DS, Honeycutt RL. 1999. Genetic evidence of DOB virus in Apodemus agrarius in Hungary. Emerg Infect Dis 5:468-470. Schmaljohn CS, Hjelle B. 1997. Hantaviruses: a global disease problem. Emerg Infect Dis 3:95-104.

Sibold C, Meisel H, Lundkvist Å, Schulz A, Cifire F, Ulrich R, Kozuch O, Labuda M, Krüger DH. 1999. Short report: simultaneous occurrence of Dobrava, Puumala, and Tula hantaviruses in Slovakia. Am J Trop Med Hyg 61:409-11.

Sibold C, Ulrich R, Labuda M, Lundkvist ̊, Martens H, Schutt M, Gerke P, Leitmeyer K, Meisel H, Krüger DH. 2001. Dobrava hantavirus causes hemorrhagic fever with renal 
For J.Med.Virol.

427 syndrome in central Europe and is carried by two different Apodemus mice species. J

428 Med Virol 63:158-167.

429 Takenaka A, Gibbs CJ, Gajdusek DC. 1985. Antiviral neutralizing antibody to Hantaan

430 virus as determined by plaque reduction technique, Arch Virol 84:197-206.

431 Trencséni T, Keleti B. 1971. Clinical aspects and epidemiology of Haemorrhagic fever

432 with renal syndrome. Analysis of the clinical and epidemiological experience in

433 Hungary. Akadémia Kiadó, Budapest, pp.247.

434 Vapalahti O, Mustonen J, Lundkvist Å, Henttonen H, Plyusnin A, Vaheri A. 2003.

435 Hantavirus infections in Europe (Review). Lancet Infect Dis 3:653-661. 


\section{Legends to figures}

Fig. 1. Rodent collecting localities in Hungary (shown as full circles). Two localities (TR16 and TR17) that are discussed in the article are marked. Stars indicate localities from where hantavirus sequences have been recovered earlier (Scharninghausen et al., 1999; Jakab et al., 2007a, 2008).

Fig. 2. Phylogenetic trees of Puumala and related hantaviruses based on (A) complete $\mathrm{S}$ segment coding sequences and (B) partial M segment sequences (nt 21612570). Numbers show the bootstrap support values for the branching points (500 replicates). Only the values higher than $70 \%$ are shown. Abbreviations: SNV, Sin Nombre virus, strain NM H10; HTNV, Hantaan virus, strain 76-118; TULV, Tula virus, strain Moravia02v; MUJV, Muju virus; HOKV, Hokkaido virus. Genetic lineages of PUUV: ALAD, Alpe-Adrian; CE, Central European; S-SCA, South Scandinavian; FIN, Finnish; RUS, Russian; N-SCA, North Scandinavian; DAN, Dannish.

Fig. 3. Phylogenetic trees of Dobrava, Saaremaa and related hantaviruses based on (A) partial M segment sequences (nt 1704-1969), and partial S segment sequences: (B) nt 377-935; (C) nt 707-935; (D) nt 410-800. Numbers show the bootstrap support values for the branching points (500 replicates). Only the values higher than $50 \%$ are shown. Abbreviations: DOBV, Dobrava virus; SAAV, Saaremaa virus; HTNV, Hantaan virus, strain 76-118 (if not specified otherwise); SEOV, Seoul virus, strain Sapporo rat (if not specified otherwise); THAIV, Thailand virus, strain741; GOUV, Gou virus, strain Nc167; SANGV, Sangassou virus strain SA14; PUUV, Puumala virus, strain Sotkamo. 